\title{
Tuning Domain Size and Crystallinity in Isoindigo/PCBM Organic Solar Cells via Solution Shearing
}

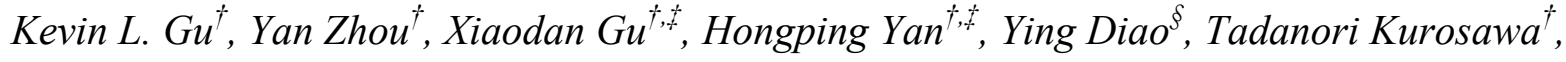
Baskar Ganapathysubramanian", Michael F. Toney", Zhenan Bao ${ }^{\dagger, *}$

${ }^{\dagger}$ Department of Chemical Engineering, Stanford University, Stanford, CA 94305, USA

${ }^{\ddagger}$ Stanford Synchrotron Radiation Lightsource, SLAC National Accelerator Laboratory, Menlo Park, CA 94025, USA

${ }^{\S}$ Department of Chemical and Biomolecular Engineering, University of Illinois at UrbanaChampaign, Urbana, IL 61801, USA

${ }^{\|}$Department of Mechanical Engineering, Iowa State University, Ames, IA 50011, USA

*Corresponding Author: zbao@stanford.edu 


\section{ABSTRACT}

Despite having achieved the long sought-after performance of $10 \%$ power conversion efficiency, high performance organic photovoltaics (OPVs) are still mostly constrained to lab scale devices fabricated by spin coating. Efforts to produce printed OPVs lag considerably behind, and the sensitivity to different fabrication methods highlights the need to develop a comprehensive understanding of the processing-morphology relationship in printing methods. Here we present a systematic experimental investigation of a model low bandgap polymer/fullerene system, polyisoindigo thienothiophene/ $\mathrm{PC}_{61} \mathrm{BM}$, using a lab-scale analogue to roll-to-roll coating as the fabrication tool in order to understand the impact of processing parameters on morphological evolution. We report that domain size and polymer crystallinity can be tuned by a factor of two by controlling the temperature and coating speed. Lower fabrication temperature simultaneously decreased the phase separation domain size and increased the relative degree of crystallinity in those domains, leading to improved photocurrent. We conclude that domain size in isoindigo/PCBM is dictated by spontaneous phase separation rather than crystal nucleation and growth. Furthermore we present a model to describe the temperature dependence of domain size formation in our system, which demonstrates that morphology is not necessarily strictly dependent on the evaporation rate, but rather on the interplay between evaporation and diffusion during the printing process.

KEYWORDS: Organic photovoltaics, isoindigo, phase separation, morphology evolution, x-ray scattering 


\section{Introduction}

Organic photovoltaics (OPVs) have attracted considerable interest during the past two decades due to their unrivaled advantages in weight, mechanical flexibility, and cost.[1] It has been well established that the efficiency of OPVs is critically dependent on the bulk heterojunction (BHJ) morphology, and recent efforts to engineer BHJs have pushed the efficiency beyond the long sought-after value of $10 \%$ power conversion efficiency (PCE).[1-4] However, these impressive devices are exclusively fabricated using spin coating, and cells prepared from more scalable methods such as blade coating and especially roll-to-roll printing tend to exhibit dramatically lower performance.[5] This suggests that despite numerous reports correlating morphology and performance, a better understanding of the effects of processing on morphology in the context of scalable coating methods is still needed.[6-8]

In spite of its popularity as a fabrication method for OPVs, [9] spin coating suffers from the inability to provide a uniform shear rate and is also difficult to adapt to high-throughput largearea fabrication.[10] Here we utilize a printing technique called solution shearing (Figure 1),[1114] which serves as a lab-scale analogue to roll-to-roll printing that enables high levels of control over processing parameters. Whereas spin coating is a radial method, solution shearing is linear method closely representing the fluid mechanics of roll-to-roll printing. Another key advantage of solution shearing as a model tool for morphology studies over spin coating is the possibility to access a wider range of controllable fabrication parameters. 
(a)

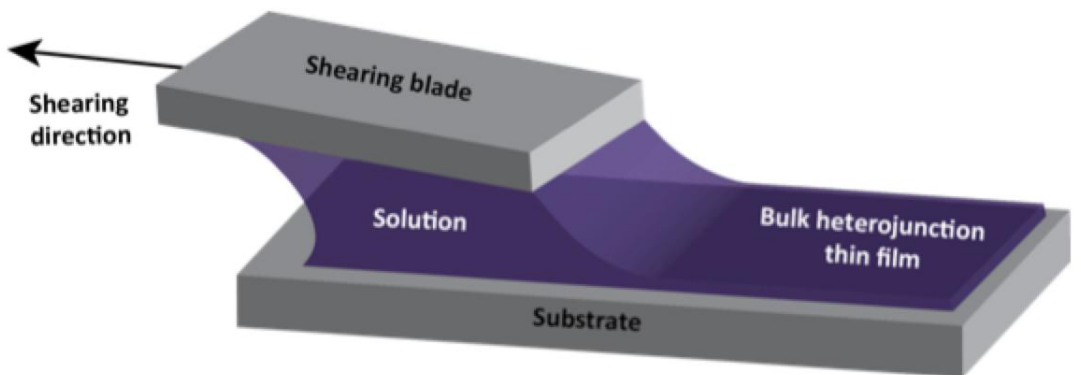

(b)

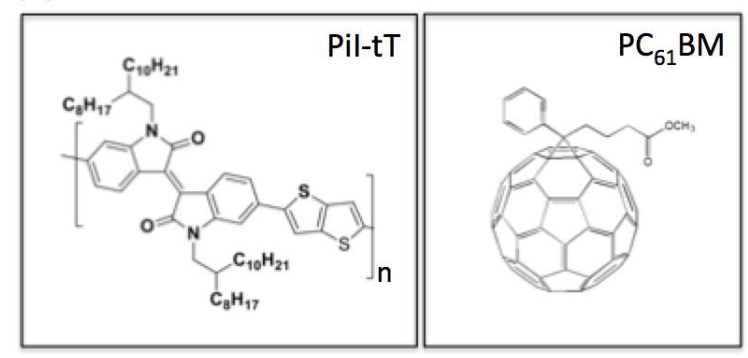

(c)

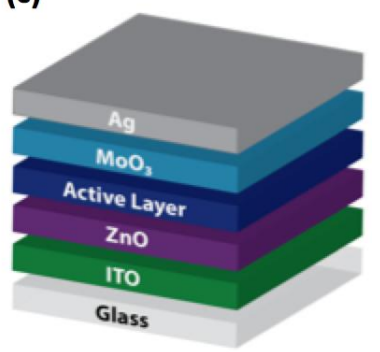

Figure 1. (a) Schematic of solution shearing setup. Chlorobenzene ink containing electron donor polymer and acceptor fullerene is held between the blade and substrate via capillary forces. As the blade translates parallel to the substrate and solvent evaporates, a solid thin film bulk heterojunction is deposited. (b) Electron donor, poly-isoindigo thienothiophene (PiI-tT), and electron acceptor, $\mathrm{PC}_{61} \mathrm{BM}$. (c) Schematic of inverted solar cell structure.

The ability to rationally control the phase separation size and crystallinity of the bulk heterojunction is crucially important to improving PCE in OPVs. It has been widely shown that obtaining desirable domain size and high crystallinity is critical for high device current.[15-21] Domains much larger than the typical exciton diffusion length of $\sim 10 \mathrm{~nm}$ for polymers result in high degrees of exciton trapping and loss of photocurrent.[17,22] A great deal of research effort has been directed at controlling morphology by methods such as tuning molecular weight, thermal annealing, choice of solvent, and the addition of processing additives.[10] However, the impact of fabrication parameters, especially for blade-coating or roll-to-roll coating, on 
morphological characteristics remains relatively unexplored until recently.[23-28] In light of the lower performance of printed OPVs,[5] it is imperative to understand the influence of processing parameters on morphology evolution, particularly in linear coating methods.

There have been experimental studies on the role of crystal nucleation/growth and spontaneous phase separation (liquid demixing of amorphous components) in the formation of P3HT/PCBM BHJs using spin coating and blade coating. Empirically it has been observed that faster spin coating speeds result in smaller phase separated domains with the assumption that fast evaporation gives less time for structure development.[29-32] However, there is debate regarding the mechanism of morphology evolution in OPV systems. Campoy-Quiles et al. have argued that morphology evolution consists of initial polymer crystallization followed by diffusion of PCBM to nucleation sites.[31] Conversely, others argue that spinodal demixing precedes crystallization, and that polymer crystallization is not the major phase separation process controlling domain size.[32] It should be noted that the majority of the above studies investigated $\mathrm{P} 3 \mathrm{HT} / \mathrm{PCBM}$ as a model system for morphology evolution,[29-32] whereas newer low bandgap polymers have not been as extensively studied in this manner. Franeker et al. reported that spinodal decomposition precedes polymer aggregation in diketopyrrolopyrrolebased polymers,[33] and explore how processing parameters influence BHJ morphology, but notably only in the context of spincoating.[34]

Here we present a systematic study of a model low-bandgap polymer/fullerene OPV system, poly isoindigo-thienothiophene (PiI-tT)/ $\mathrm{PC}_{61} \mathrm{BM}$, using solution shearing as the coating method in order to understand influence of processing conditions on morphology development. Even though PiI-tT is not the most efficient donor polymer, the understanding and methodology gained can still be applied to other systems. In varying the fabrication temperature and solution 
shearing speed, we observed a nearly twofold difference in polymer crystallinity and phase separated domain size. We determine that domain size in this system is determined by spontaneous phase separation as opposed to crystallite nucleation and growth. Furthermore, experimental results of processing temperature vs domain size correlate well with previously reported computational simulations. Wodo et al. have computationally predicted that phase separation is highly dependent on the mass Biot number, which quantifies the relative importance of evaporation to diffusion.[35] Here we present a model to describe the fundamental temperature dependence of domain size formation in our system, demonstrating that morphology is dictated by the interplay between evaporation and diffusion during the drying process.

\section{Results}

In order to correlate device performance with morphology data, we investigated samples fabricated at different temperatures but with constant active layer thicknesses. Herein, the internal electric field and active layer absorption within each series are identical. Fabrication temperature was varied from 35 to $95{ }^{\circ} \mathrm{C}$ and a series of 5 cells was fabricated with equal active layer thickness of $44 \mathrm{~nm}( \pm 7 \mathrm{~nm})$. The constant thickness was obtained by empirically tuning the shearing speed between 0.01 and $1.0 \mathrm{~mm} / \mathrm{s}$. In the evaporation deposition regime, increasing temperature or decreasing shearing speed leads to thicker films due to more solvent evaporation per unit length.[36] Similarly, a second thicker series of 5 samples was fabricated at the same temperatures (at slower shearing speeds), resulting in films of $92 \mathrm{~nm}( \pm 8 \mathrm{~nm})$. These two series are hereafter referred to as the "Thin series" and "Thick series," respectively (refer to Table 1 for parametric details). In order to develop an understanding of the phenomena governing morphology evolution in isoindigo/PCBM, samples were characterized for UV-vis absorption, 
photovoltaic device performance, phase separation domain size, and relative degree of crystallinity.

\subsection{Photophysical properties}

Figure 2 shows the UV-vis absorption spectra of the two thickness series, normalized to the primary donor polymer absorption peak at $720 \mathrm{~nm}$. This peak originates from the optical band gap of PiI-tT $(1.6 \mathrm{eV})$. Interestingly we find that the composition of the film is thickness dependent, with thicker films containing more PCBM as evidenced by the relative absorption intensities at $720 \mathrm{~nm}$ (donor polymer) and $400 \mathrm{~nm}$ (acceptor PCBM) in Figure 2a and b.

The higher PCBM content in thicker films suggests that the different diffusivities of the donor and acceptor materials have a profound effect on the final film composition in the evaporative deposition regime. This compositional thickness dependence is not observed in similar spin coated films (Figure 2c). To the best of our knowledge this processing-dependent composition has not been reported previously. We hypothesize that the observed composition change with thickness is due to the differential diffusivities of the polymer and PCBM molecules in the context of the Soret effect.[37] Evaporative cooling at the drying front induces a thermal gradient, which may cause PCBM to preferentially diffuse from the solution reservoir resulting in higher PCBM content in the final film. In thicker films, a slower shearing speed increases film drying time, increasing the magnitude of the effect. 

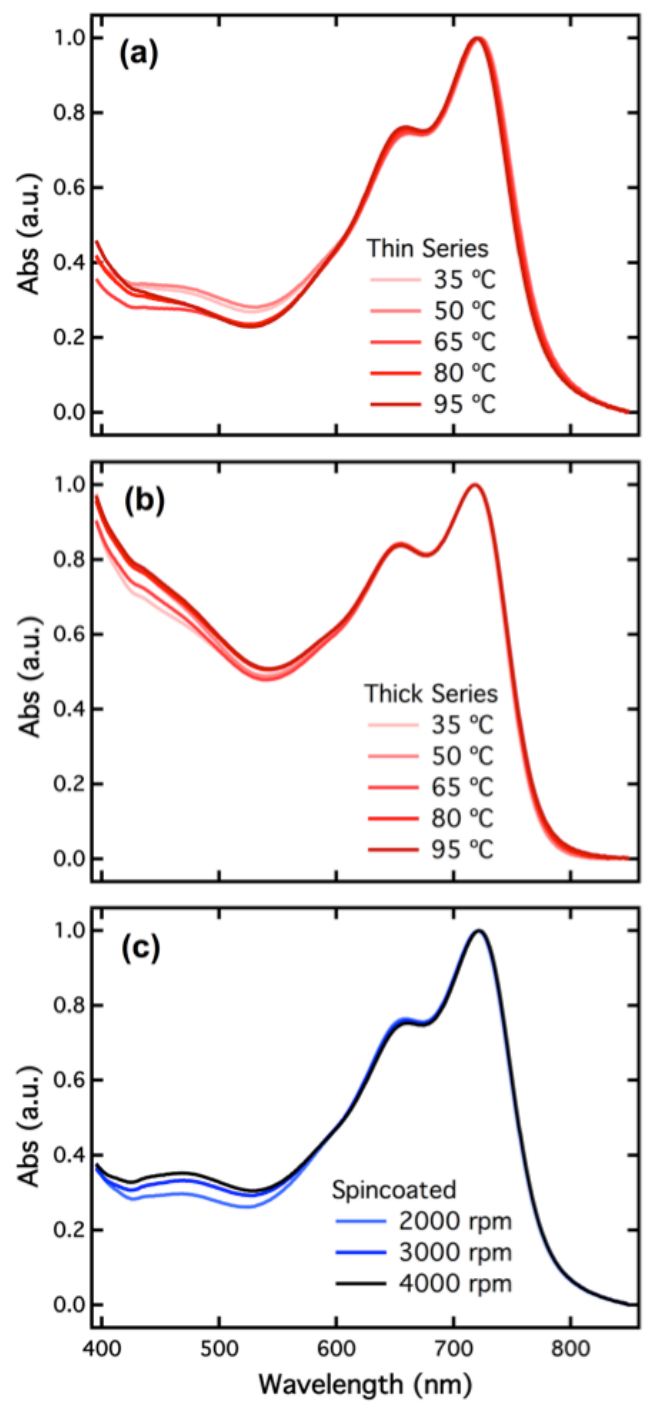

Figure 2. UV-vis absorption spectra of the Thin film series (a) and Thick film series (b) of polyisoindigo-thienothiophene $/ \mathrm{PC}_{61} \mathrm{BM} \mathrm{BHJ}$ films. Fabrication temperature and shearing speed were varied concurrently to maintain a constant thickness within each series. All absorption intensities have been normalized to the primary donor absorption peak at $720 \mathrm{~nm}$ (a-c). The relative intensities at $720 \mathrm{~nm}$ and $400 \mathrm{~nm}$ indicate that polymer/PCBM composition is thickness dependent (between (a) and (b)), while composition within each thickness series remains constant (within (a) or (b)). (c) This behavior is in contrast to spin coated BHJs, in which the 
composition is identical between film thicknesses of $60-80 \mathrm{~nm}$, spin coated at speeds of 2000 rpm -4000 rpm.

Strikingly, composition is constant for each given thickness even as temperature and shearing speed are varied simultaneously. To obtain equal film thickness within a series, coating is done at either a higher substrate temperature at a higher coating speed, or a lower substrate temperature at a lower coating speed. In each case, the result is a constant composition for all films in a thickness series due to a constant deposition rate per unit length. These results highlight a major difference between solution shearing and spin coating. There is no solution reservoir during spin coating and therefore the final film composition is unchanged from the initial solution composition. In the context of the present study, we can conclude that any differences in cell performance, domain size, and crystallinity between films within each thickness series are not due to differences in composition.

\subsection{Solar cell device performance}

Solar cell devices are prepared here in order to understand the relationship between performance characteristics and morphology, rather than achieving the most efficient solar cells. The highest reported efficiency achieved for PiI-tT/PCBM is 1.74\%.[38] For films prepared at higher temperatures in both thickness series, the PCE decreased by about a factor of 2 in each case (Table 1). The dominant component of PCE we observed to contribute to the variation in performance was the short circuit current $\left(J_{\mathrm{SC}}\right)$, which decreased by nearly a factor of two with increasing fabrication temperature. The open circuit voltage $\left(V_{\mathrm{OC}}\right)$ and fill factor $(\mathrm{FF})$ showed 
relatively little variation with respect to the photocurrent. The Thick series showed higher current when compared to the Thin series due to increased absorption by the thicker films.

Table 1. Solar cell performance at various shearing speed $(v)$ and temperature (T) for Thin series cells (top) and Thick series cells (bottom). PCE varies primarily due to photocurrent as $J_{\mathrm{SC}}$ decreases by nearly a factor of 2 with increasing temperature in both thickness series. $V_{\mathrm{OC}}$ and FF remain relatively constant in comparison to $J_{\mathrm{SC}}$ within each series.

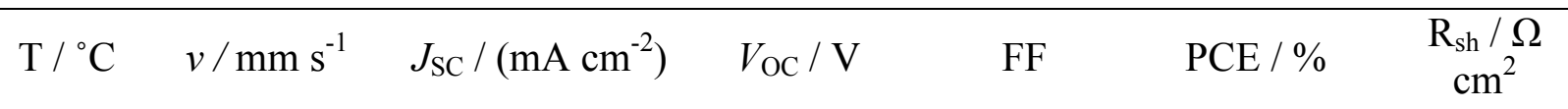

\begin{tabular}{ccccccc}
\hline \multicolumn{2}{l}{ Thin Series $(44 \mathrm{~nm})$} & & & & & \\
35 & 0.10 & $-5.08 \pm 0.35$ & $0.82 \pm 0.01$ & $0.54 \pm 0.03$ & $2.24 \pm 0.08$ & 7 \\
50 & 0.22 & $-3.69 \pm 0.18$ & $0.79 \pm 0.00$ & $0.52 \pm 0.01$ & $1.52 \pm 0.11$ & 6 \\
65 & 0.41 & $-3.74 \pm 0.36$ & $0.80 \pm 0.03$ & $0.52 \pm 0.01$ & $1.58 \pm 0.21$ & 8 \\
80 & 0.66 & $-3.78 \pm 0.35$ & $0.82 \pm 0.01$ & $0.53 \pm 0.01$ & $1.64 \pm 0.12$ & 11 \\
95 & 0.99 & $-2.73 \pm 0.58$ & $0.84 \pm 0.01$ & $0.52 \pm 0.04$ & $1.18 \pm 0.26$ & 12
\end{tabular}

Thick Series $(92 \mathrm{~nm})$

$\begin{array}{ccccccc}35 & 0.03 & -5.75 \pm 0.93 & 0.79 \pm 0.01 & 0.45 \pm 0.01 & 2.06 \pm 0.37 & 17 \\ 50 & 0.08 & -4.51 \pm 0.19 & 0.82 \pm 0.00 & 0.47 \pm 0.01 & 1.75 \pm 0.06 & 10 \\ 65 & 0.14 & -4.23 \pm 0.23 & 0.83 \pm 0.00 & 0.54 \pm 0.02 & 1.89 \pm 0.15 & 9 \\ 80 & 0.22 & -3.87 \pm 0.34 & 0.84 \pm 0.00 & 0.53 \pm 0.02 & 1.71 \pm 0.19 & 12 \\ 95 & 0.33 & -3.13 \pm 0.26 & 0.84 \pm 0.02 & 0.50 \pm 0.02 & 1.33 \pm 0.10 & 24\end{array}$




\subsection{Domain size characterization}

Resonant Soft X-ray Scattering (RSoXS) was used to characterize the domain size in both series of BHJ films. The characteristic peak in the intensity times the square of the scattering vector vs scattering vector plot represents the period of scatterers, which may be used as an indication of the average phase separation domain size in the bulk heterojunction (Figure 3).[18,39-41] The x-ray energy was scanned from 270 to $290 \mathrm{eV}$ through the aromatic $\mathrm{C} 1 \mathrm{~s}-\pi^{*}$ resonance to maximize the scattering intensity, which was determined to be at $284 \mathrm{eV}$.
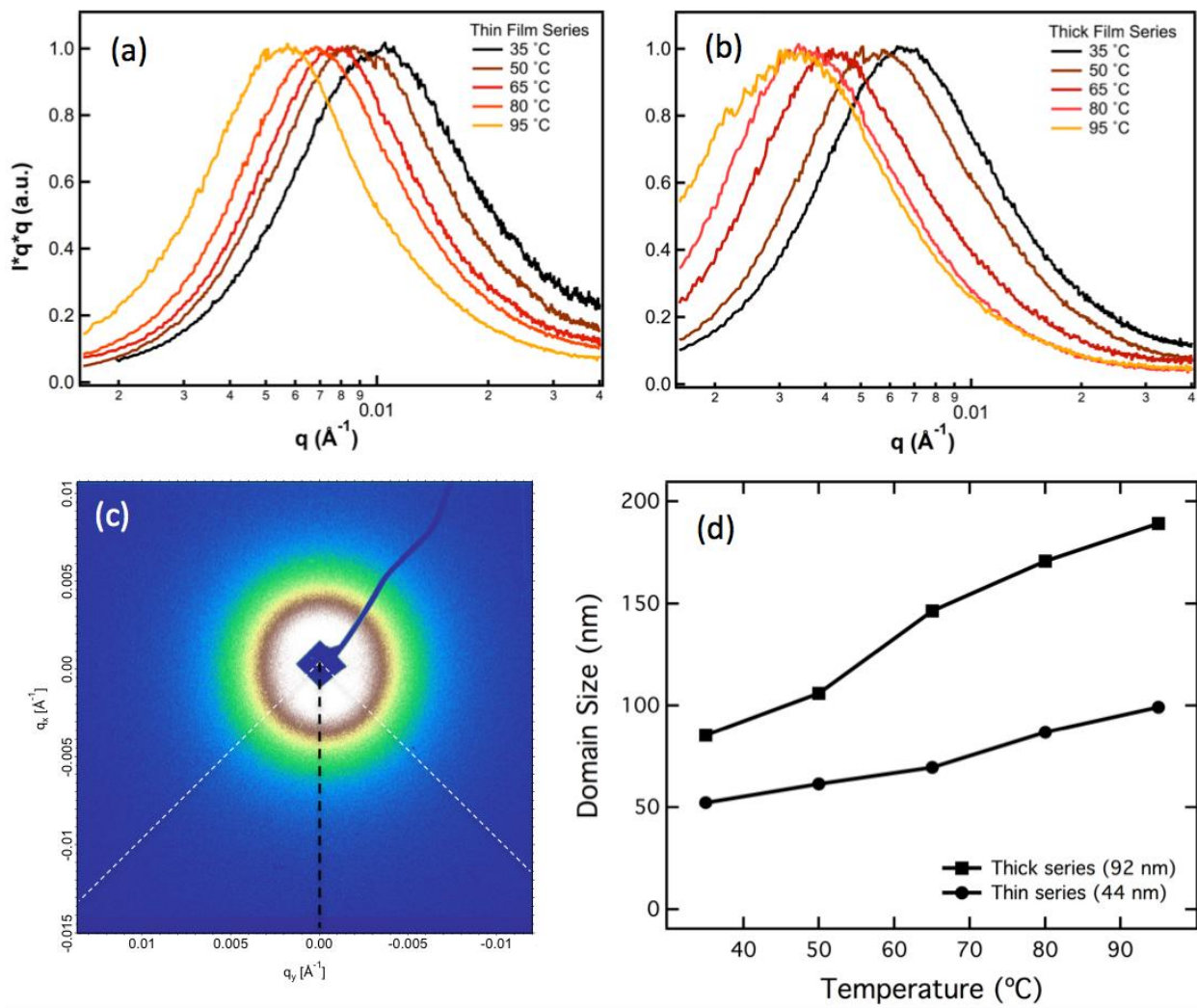

Figure 3. $(a, b)$ Intensity times the square of the scattering vector vs scattering vector for Thin and Thick film series, normalized to the peak value. 1D RSoXS sector averages for were calculated from 2D scattering patterns as shown in Figures S1 and S2. (c) Representative isotropic $2 \mathrm{D}$ scattering profile $\left(35^{\circ} \mathrm{C}, 0.10 \mathrm{~mm} / \mathrm{s}\right)$ and corresponding sector average. (d) Phase 
separation domain size as a function of coating temperature. The average domain size is calculated by $2 \pi / q_{\text {peak. }}$. In both series, increasing fabrication temperature results in increased domain size.

The RSoXS data show a clear trend that smaller domains formed at lower temperatures. At higher fabrication temperature, domain sizes were up to a factor of two greater in both thickness series between 35 and $95{ }^{\circ} \mathrm{C}$. Correlating domain size with solar cell performance, we found that the short circuit current was significantly lower in cells with larger domains (Figure 4a). This agrees with the conventionally accepted picture that domain sizes larger than the exciton diffusion length decreases the fraction of excitons able to diffuse to donor/acceptor interfaces, leading to a decrease in photocurrent due to exciton trapping and geminate recombination in large domains.[2]

It should be noted that although temperature and shearing speed are concurrently tuned within each thickness series in order to maintain the same thickness, the increase in domain size is due to the increase in temperature rather than the increase in shearing speed. This is evident by comparing cells fabricated at different speeds at the same temperature, from which we observed that increasing shearing speed strictly decreased domain size (Figure 4b). This result may be due to shear-induced nucleation, where polymer chains are extended due to extensional flow during the shearing motion of the fluid.[23] However, this effect was not investigated in detail in the present study. 

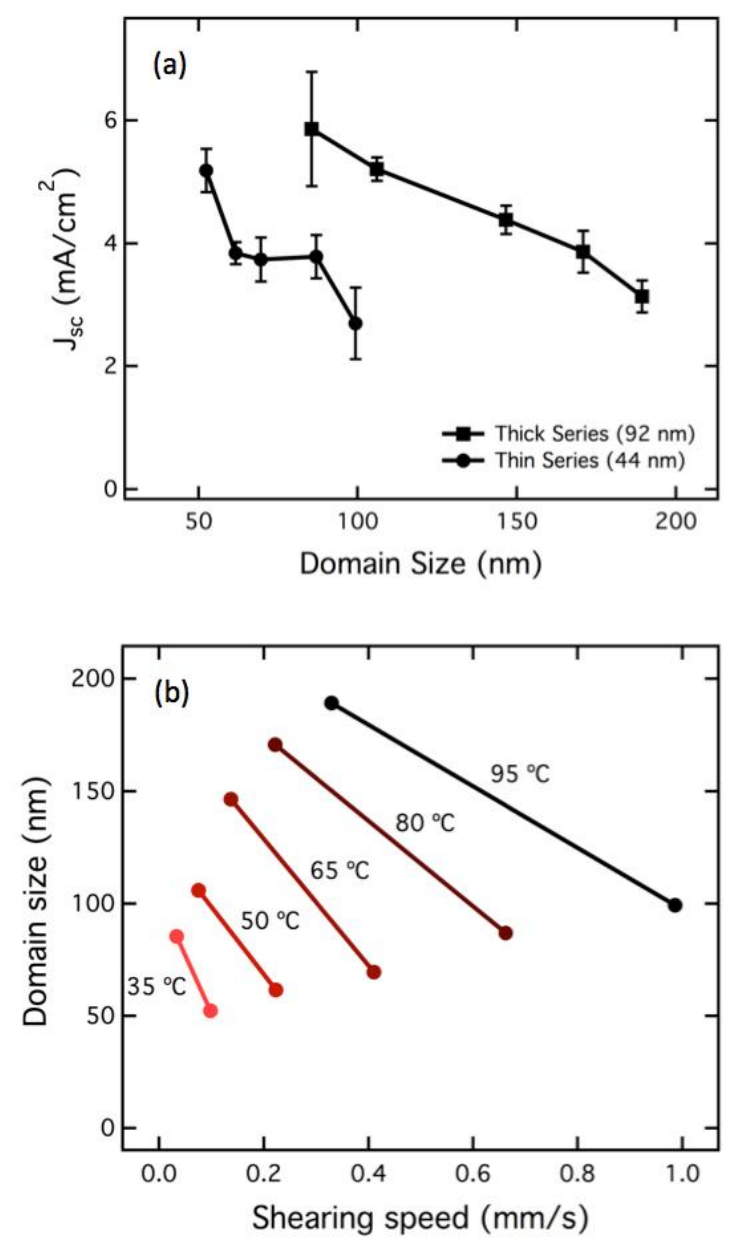

Figure 4. (a) Short circuit device current decreases with increasing domain size (error bars represent one standard deviation for 6-10 measured cells). Exciton diffusion to donor/acceptor interfaces is reduced in larger phase separated domains, resulting in larger recombination losses. (b) Domain size as a function of coating speed. Pairs of cells were fabricated at different coating speeds at the same temperature, indicating that coating speed can be used to tune domain size.

\subsection{Relative Degree of Crystallinity}

In order to quantify the crystallinity of the films, grazing-incidence x-ray diffraction (GIXD) was performed at SLAC SSRL Beamline 11-3. The relative degree of crystallinity (RDoC) was extracted following procedures described in previous works.[23,42-44] Pole figures of the (200) 
polymer lamellar stacking peak were integrated after a geometrical correction was applied to account for crystallites at a particular orientation. The polar angle describes the relative orientation of crystallites with respect to the substrate, with $\chi=0^{\circ}$ and $\chi=90^{\circ}$ representing faceon and edge-on crystallites, respectively. After normalization by irradiated volume, background subtraction, and multiplication by a geometrical correction factor, the total integrated area from $\chi$ $=\left[-84^{\circ}, 84^{\circ}\right]$ represents the relative degree of crystallinity.[23] Similar analysis was performed for PCBM relative degree of crystallinity. The nanoscale PCBM crystallites are clearly observed as the isotropic ring at $1.36 \AA^{-1}$ in the GIXD pattern.[45] From scans parallel and perpendicular to the shearing direction, we deduced isotropic in-plane orientation of crystalline domains to warrant applicability of the geometrical correction.

We performed this analysis on the Thick series, revealing that $\mathrm{RDoC}$ of both the polymer and PCBM decreased at higher temperatures by more than a factor of two (Figure 5). Decreased crystallinity implies that the polymer phase of the BHJ becomes significantly more disordered at higher fabrication temperatures. Higher crystallinity at lower temperatures may be explained by longer crystal growth time, which is consistent with previous reports.[46] In solution shearing, increasing temperature while concurrently increasing shearing speed, to maintain constant film thickness, decreases the evaporation time per unit length, giving polymer crystallites less time to grow into ordered structures despite the lower energetic barrier to nuclei formation.

Device performance also correlates well with RDoC. Higher temperature produced cells with lower crystallinity, which may contribute to the decreased $J_{S C}$. It has been argued that higher crystallinity may increase the exciton diffusion length[19-21] and charge carrier mobility[4] while reducing non-geminate charge carrier recombination,[2,47-49] resulting in improved $J_{S C}$. 

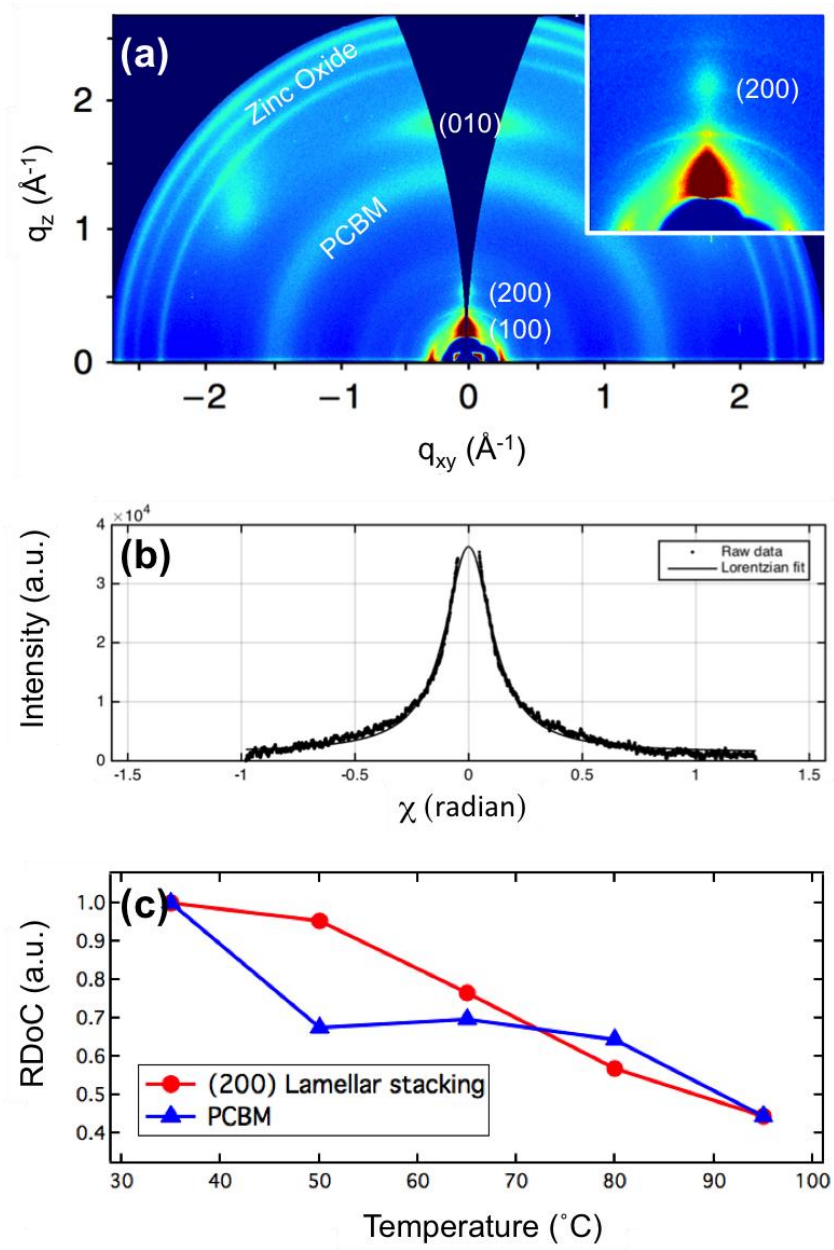

Figure 5. (a) Representative $2 \mathrm{D}$ grazing-incidence $\mathrm{x}$-ray diffraction pattern of a BHJ thin film $\left(35{ }^{\circ} \mathrm{C}, 0.1 \mathrm{~mm} \mathrm{~s}^{-1}\right)$. The three outer rings result from the $\mathrm{ZnO}$ interlayer between the Si substrate and active layer. The polymer $\pi-\pi$ stacking peak (010) and the lamellar peaks (100) and (200) are labeled. (Inset) Magnified images of the (200) peak (without geometrical correction for clarity). (b) Pole figure of the polymer (200) lamellar stacking peak, where the polar angle $\chi$ indicates the crystallite orientation. (c) Relative degree of crystallinity for polymer (red) and PCBM (blue) vs shearing temperature for the Thick sample series. The corrected intensity of the peaks, or $|\sin (\chi)| * I(\chi)$, represents the relative population of the crystallites with a particular orientation $\chi$, where $\chi=0^{\circ}$ indicates face-on orientation and $|\chi|=\pi / 2$ indicates edge-on 
orientation. $\mathrm{RDoC}$ was obtained by integrating the area of the geometrically corrected pole figures.

\section{Discussion}

From the morphological results we gained insights into which physical phenomenon primarily drives morphology evolution. To understand whether spontaneous phase separation or crystallization determines domain size in our isoindigo/fullerene system, we note the two competing trends of crystallinity and domain size. In the case of crystallite growth-dominated domain size, one would expect crystallinity and domain size to be positively correlated. Crystallites would nucleate and grow in solution and would aggregate upon solvent evaporation. This is in contrast to spontaneous phase separation, which after solvent evaporation results in a kinetically trapped morphology irrespective of crystallinity.

At higher temperatures, polymer crystallinity was significantly reduced, and the inverse correlation between domain size and both polymer and PCBM crystallinity suggests that domain size in our system is primarily dictated by spontaneous phase separation rather than crystal nucleation and growth. This initial phase separation behavior provides indirect evidence of the immiscibility or partial miscibility of PiI-tT and PCBM. We extend this observation further and argue that the relative effects of evaporation and diffusion determine the degree to which phase separation can occur, which in turn dictates domain size in the final dried film. We present the following model of domain size formation.

To describe the spontaneous phase separation in OPV systems it is necessary to consider both external solvent evaporation and internal diffusion within the liquid film during drying. The mass Biot number $\left(B i_{m}\right)$ is a dimensionless quantity that describes the ratio of the rate of evaporation 
to the rate of diffusion. High $B i_{m}$ indicates that evaporation dominates over diffusion, and vice versa. Wodo et al. have computationally predicted that higher $B i_{m}$ leads to smaller domains via a continuum model of the drying dynamics of a general OPV system.[35] Several studies have attributed smaller phase separated domains to faster evaporation rates in that there is less time for structure development.[29-32] In our case, the evaporation rate increases with temperature and yet we observed that higher temperature led to larger domains. Therefore, diffusion must also play an important role on the domain size. Although data on diffusivity coefficients for OPV materials in solution is very limited due to the lack of suitable measurement techniques,[37] there is still much insight to be gained from a qualitative understanding of the interplay between diffusion and evaporation. Here, we derive the temperature dependence of $B i_{m}$ to account for diffusion.

To relate temperature to $B i_{m}$, we perform an energy balance and determine that $B i_{m}$ decreases as temperature increases for chlorobenzene as the solvent. We present the following expression to describe the temperature dependence of $B i_{m}$ in general:

$$
B i_{m}(T) \sim T * \exp \left[\frac{E_{a}}{R T}-\left(A-\frac{B}{T+C}\right)\right]
$$

where $E_{a}=0.075 \mathrm{~J} \mathrm{~mol}^{-1}$ is the experimentally extracted Arrhenius activation energy for diffusion, $A=13.9, B=3174.8 \mathrm{~K}$, and $C=211.7 \mathrm{~K}$ are the Antoine coefficients for chlorobenzene,[50] $R$ is the gas constant, and $T$ is the absolute temperature in Kelvin. For a full derivation of Equation 1, we refer the reader to the Supporting Information. Due to the lack of individual component diffusivity data, the diffusivity for chlorobenzene is used here as a proxy for overall diffusion within the system. We show that $B i_{m}$ is a monotonically decreasing function 
of temperature for the range investigated with $B i_{m}\left(35{ }^{\circ} \mathrm{C}\right)=0.13$ and $B i_{m}\left(95{ }^{\circ} \mathrm{C}\right)=0.08$. This suggests that as temperature is increased, the relative effects of diffusion become more prominent versus evaporation, increasing the degree of phase separation prior to complete solidification. Our experimental results also match well with computational predictions as described by Wodo et al. [35] in that higher temperature (decreasing $B i_{m}$ ) causes the formation of larger domains. These results suggest that the formation of large domains in isoindigo/PCBM is primarily dictated by enhanced diffusion within the drying film via spontaneous phase separation.

\section{Conclusion}

The extreme sensitivity of high performance OPV materials to different fabrication methods highlights the need to develop a fundamental understanding of the influence of processing parameters on morphology evolution. To this end, we have studied a model low bandgap polymer/fullerene OPV system using solution shearing coating, a lab-scale analogue to roll-toroll printing. With solution shearing we are able to independently tune substrate temperature and coating speed, whereas in spin coating often the substrate and solution can only be preheated. Additionally in contrast to spin coating, we observed that composition in shear-coated films is thickness dependent due to solute diffusion from the solution reservoir. Our experimental results show that higher fabrication temperature while maintaining the same BHJ thickness results in larger domain size and decreased crystallinity, indicating that the morphology is dominated by spontaneous phase separation rather than crystal nucleation and growth.

We have provided a temperature dependence equation for the mass Biot number, and we anticipate that our model may be applicable to similar OPV BHJ systems with fullerene and 
potentially other polymer/small molecule systems. Further experiments are warranted to investigate the extent of general applicability to other potentially high performance systems. The ability to rationally design morphology is crucial to producing ever higher-performing cells, and these results indicate that printed polymer/fullerene BHJs need to be tuned carefully due to the sensitivity of domain size and crystallinity to coating conditions. Our results emphasize that controlling fabrication parameters of linear coating methods is a powerful tool and essential consideration for tuning morphology in polymer/fullerene OPVs.

\section{Experimental Section}

Materials: Poly isoindigo thienothiophene (PiI-tT) $\left(\mathrm{Mn}=16,100 \mathrm{~g} \mathrm{~mol}^{-1}\right)$ was synthesized according to previously reported procedures and purified via size-exclusion chromatography with HPLC-grade chloroform as solvent.[17,51-53] Phenyl-C61-butyric acid methyl ester $\left(\mathrm{PC}_{61} \mathrm{BM}\right)$ and anhydrous chlorobenzene were purchased from Sigma Aldrich and used as received. Glass substrates coated with indium-doped tin oxide (ITO) (sheet resistance of $13 \Omega / \square$ ) were purchased from Xin Yan Technology Lt. Silicon wafers for GIXD were purchased from University Wafer. Silicon nitride $\left(\mathrm{Si}_{3} \mathrm{~N}_{4}\right)$ windows purchased from Norcada Inc. were used for RSoXS transmission measurements. Solution Shearing: The shearing solution was prepared by dissolving $10 \mathrm{mg}$ polymer and $15 \mathrm{mg}$ fullerene per milliliter of chlorobenzene. After stirred heating at $80{ }^{\circ} \mathrm{C}$ for $10 \mathrm{~h}$, the solution was passed through a $0.45 \mu \mathrm{m}$ PTFE filter to remove large aggregates and contaminants. The sample substrate and top shearing blade were held in position by vacuum with a separation distance of $30 \pm 5 \mu \mathrm{m}$. Approximately $10 \mu \mathrm{L} \mathrm{cm}^{-2}$ of solution was injected between the substrate and shearing blade by pipette and held in place by capillary forces immediately prior to shearing. The top blade was translated at a speed of $0.03-1.0 \mathrm{~mm} \mathrm{~s}^{-1}$ as a 
thermoelectric heater stage maintained temperature $\left( \pm 2{ }^{\circ} \mathrm{C}\right)$ during shear coating. Solar Cell Fabrication and Testing: Inverted geometry devices were fabricated on ITO-patterned glass substrates. Glass/ITO substrates were subjected to UV-ozone cleaning for 20 min immediately prior to use. A zinc oxide sol gel solution was immediately spin coated on the top surface (5000 rpm for $30 \mathrm{sec}$ ) and baked for $30 \mathrm{~min}$ at $200{ }^{\circ} \mathrm{C}$ in air to form a $30 \mathrm{~nm} \mathrm{ZnO}$ layer.[54] After shearing of the active layer at various temperatures and shear speeds, anode materials $(15 \mathrm{~nm}$ $\mathrm{MoO}_{3}$ and $\left.150 \mathrm{~nm} \mathrm{Ag}\right)$ were thermally evaporated in high vacuum $\left(1 \times 10^{-5}\right.$ Torr $)$ to produce solar cells of $4 \mathrm{~mm}^{2}$ active area. For testing of performance metrics, devices were illuminated under a simulated AM 1.5G solar spectrum (Newport Solar Simulator 94021A) calibrated to a KG5 Si photodiode and measured by a Keithley 2400 analyzer. Solution Sheared Film Characterizations: The thicknesses of the BHJ active layers were measured on a Bruker Dektak 150 profilometer. UV-vis optical characterization of the active layer films was carried out on an Agilent Cary 6000i UV/Vis/NIR spectrometer in single-pass geometry. 2D grazing-incidence $\mathrm{x}$ ray diffraction (GIXD) patterns were collected at an incidence angle of $0.12^{\circ}$ at Beamline $11-3$ of the Stanford Synchrotron Radiation Lightsource at SLAC National Accelerator Laboratory. Resonant soft x-ray scattering (RSoXS) was performed at Beamline 11.0.1.2 at the Advanced Light Source at Lawrence Berkeley National Laboratory.[55] Films for RSoXS were fabricated on $\mathrm{Si} /$ polystyrene sulfonate and then float transferred in DI water to $\mathrm{Si}_{3} \mathrm{~N}_{4}$ windows for transmission measurements. 


\section{ASSOCIATED CONTENT}

Supporting Information. 2D RSoXS patterns for Thick and Thin series, derivation of Mass Biot number temperature dependence.

\section{ACKNOWLEDGMENTS}

X.G., Y.D., M.F.T., and Z.B. acknowledge support by the Department of Energy, Office of Energy Efficiency \& Renewable Energy, Bridging Research Interactions through the collaborative Development Grants in Energy (BRIDGE) program under Contract No. DE-FOA0000654-158. Y.Z., T.K., and Z.B. acknowledge the Office of Naval Research under Award No. N00014-14-1-0142. H.Y. acknowledges the National Science Foundation Materials Genome Initiative under Grant No. 1434799. B.G. acknowledges partial financial support from the National Science Foundation under Grant no. 1435587 and 1149365 . K.L.G. was supported by the Department of Defense (DoD) through the National Defense Science \& Engineering Graduate Fellowship (NDSEG) Program. Portions of this work were carried out at Beamline 11.0.1.2 at the Advanced Light Source, which is supported by the Director, Office of Science, Office of Basic Energy Sciences, of the U.S. Department of Energy under Contract No. DEAC02-05CH11231, and at Beamline 11-3 of the Stanford Synchrotron Radiation Lightsource, SLAC National Accelerator Laboratory, which is supported by the U.S. Department of Energy, Office of Science, Office of Basic Energy Sciences under Contract No. DE-AC02-76SF00515. 


\section{REFERENCES}

[1] G. Li, R. Zhu, Y. Yang, Polymer solar cells, Nat. Photonics. 6 (2012) 153-161. doi:10.1038/nphoton.2012.11.

[2] N.D. Treat, M.L. Chabinyc, Phase separation in bulk heterojunctions of semiconducting polymers and fullerenes for photovoltaics., Annu. Rev. Phys. Chem. 65 (2014) 59-81. doi:10.1146/annurev-physchem-040513-103712.

[3] Y. Huang, E.J. Kramer, A.J. Heeger, G.C. Bazan, Bulk heterojunction solar cells: morphology and performance relationships., Chem. Rev. 114 (2014) 7006-7043. doi:10.1021/cr400353v.

[4] Y. Liu, J. Zhao, Z. Li, C. Mu, W. Ma, H. Hu, K. Jiang, H. Lin, H. Ade, H. Yan, Aggregation and morphology control enables multiple cases of high-efficiency polymer solar cells., Nat. Commun. 5 (2014) 5293. doi:10.1038/ncomms6293.

[5] R. Søndergaard, M. Hösel, D. Angmo, T.T. Larsen-Olsen, F.C. Krebs, Roll-to-roll fabrication of polymer solar cells, Mater. Today. 15 (2012) 36-49. doi:10.1016/S13697021(12)70019-6.

[6] F.C. Krebs, Fabrication and processing of polymer solar cells: A review of printing and coating techniques, Sol. Energy Mater. Sol. Cells. 93 (2009) 394-412. doi:10.1016/j.solmat.2008.10.004.

[7] R.R. Søndergaard, M. Hösel, F.C. Krebs, Roll-to-Roll fabrication of large area functional organic materials, J. Polym. Sci. Part B Polym. Phys. 51 (2013) 16-34. doi:10.1002/polb.23192. 
[8] Y. Liu, T.T. Larsen-Olsen, X. Zhao, B. Andreasen, R.R. Søndergaard, M. Helgesen, K. Norrman, M. Jørgensen, F.C. Krebs, X. Zhan, All polymer photovoltaics: From small inverted devices to large roll-to-roll coated and printed solar cells, Sol. Energy Mater. Sol. Cells. 112 (2013) 157-162. doi:10.1016/j.solmat.2013.01.025.

[9] J. Peet, M.L. Senatore, A.J. Heeger, G.C. Bazan, The Role of Processing in the Fabrication and Optimization of Plastic Solar Cells, Adv. Mater. 21 (2009) 1521-1527. doi:10.1002/adma.200802559.

[10] C.R. McNeill, Morphology of all-polymer solar cells, Energy Environ. Sci. 5 (2012) 5653. doi:10.1039/c2ee03071c.

[11] Y. Diao, L. Shaw, Z. Bao, S.C.B. Mannsfeld, Morphology control strategies for solutionprocessed organic semiconductor thin films, Energy Environ. Sci. 7 (2014) 2145-2159. doi:10.1039/C4EE00688G.

[12] H. a. Becerril, M.E. Roberts, Z. Liu, J. Locklin, Z. Bao, High-performance organic thinfilm transistors through solution-sheared deposition of small-molecule organic semiconductors, Adv. Mater. 20 (2008) 2588-2594. doi:10.1002/adma.200703120.

[13] Y. Diao, B.C.-K. Tee, G. Giri, J. Xu, D.H. Kim, H. a Becerril, R.M. Stoltenberg, T.H. Lee, G. Xue, S.C.B. Mannsfeld, Z. Bao, Solution coating of large-area organic semiconductor thin films with aligned single-crystalline domains., Nat. Mater. 12 (2013) 665-71. doi:10.1038/nmat3650.

[14] G. Giri, S. Park, M. Vosgueritchian, M.M. Shulaker, Z. Bao, High-Mobility, Aligned Crystalline Domains of TIPS-Pentacene with Metastable Polymorphs Through Lateral 
Confinement of Crystal Growth, Adv. Mater. 26 (2014) 487-493. doi:10.1002/adma.201302439.

[15] Y. Sun, G.C. Welch, W.L. Leong, C.J. Takacs, G.C. Bazan, A.J. Heeger, Solutionprocessed small-molecule solar cells with 6.7\% efficiency., Nat. Mater. 11 (2012) 44-8. doi:10.1038/nmat3160.

[16] D.H. Kim, A.L. Ayzner, A.L. Appleton, K. Schmidt, J. Mei, M.F. Toney, Z. Bao, Comparison of the photovoltaic characteristics and nanostructure of fullerenes blended with conjugated polymers with siloxane-terminated and branched aliphatic side chains, Chem. Mater. 25 (2013) 431-440. doi:10.1021/cm303572d.

[17] Y. Zhou, T. Kurosawa, W. Ma, Y. Guo, L. Fang, K. Vandewal, Y. Diao, C. Wang, Q. Yan, J. Reinspach, J. Mei, A.L. Appleton, G.I. Koleilat, Y. Gao, S.C.B. Mannsfeld, A. Salleo, H. Ade, D. Zhao, Z. Bao, High performance all-polymer solar cell via polymer side-chain engineering, Adv. Mater. 26 (2014) 3767-72. doi:10.1002/adma.201306242.

[18] B.A. Collins, Z. Li, J.R. Tumbleston, E. Gann, C.R. McNeill, H. Ade, Absolute Measurement of Domain Composition and Nanoscale Size Distribution Explains Performance in PTB7:PC 71 BM Solar Cells, Adv. Energy Mater. 3 (2013) 65-74. doi:10.1002/aenm.201200377.

[19] H. Najafov, B. Lee, Q. Zhou, L.C. Feldman, V. Podzorov, Observation of long-range exciton diffusion in highly ordered organic semiconductors., Nat. Mater. 9 (2010) 938943. doi:10.1038/nmat2872.

[20] R.R. Lunt, J.B. Benziger, S.R. Forrest, Relationship between Crystalline Order and 
Exciton Diffusion Length in Molecular Organic Semiconductors, Adv. Mat. 22 (2010) 1233-1236. doi:10.1002/adma.200902827.

[21] M. Sim, J. Shin, C. Shim, M. Kim, S.B. Jo, J.-H. Kim, K. Cho, Dependence of Exciton Diffusion Length on Crystalline Order in Conjugated Polymers, J. Phys. Chem. C. 118 (2014) 760-766. doi:10.1021/jp409776s.

[22] Y. Kim, S. Cook, S.M. Tuladhar, S.A. Choulis, J. Nelson, J.R. Durrant, D.D.C. Bradley, M. Giles, I. McCulloch, C.-S. Ha, M. Ree, A strong regioregularity effect in selforganizing conjugated polymer films and high-efficiency polythiophene:fullerene solar cells, Nat. Mater. 5 (2006) 197-203. doi:10.1038/nmat1574.

[23] Y. Diao, Y. Zhou, T. Kurosawa, L. Shaw, C. Wang, S. Park, Y. Guo, J. a. Reinspach, K. Gu, X. Gu, B.C.K. Tee, C. Pang, H. Yan, D. Zhao, M.F. Toney, S.C.B. Mannsfeld, Z. Bao, Flow-enhanced solution printing of all-polymer solar cells, Nat. Commun. 6 (2015) 7955. doi:10.1038/ncomms8955.

[24] S. Kouijzer, J.J. Michels, M. van den Berg, V.S. Gevaerts, M. Turbiez, M.M. Wienk, R.A.J. Janssen, Predicting Morphologies of Solution Processed Polymer:Fullerene Blends, J. Am. Chem. Soc. 135 (2013) 12057-12067. doi:10.1021/ja405493j.

[25] K.W. Chou, B. Yan, R. Li, E.Q. Li, K. Zhao, D.H. Anjum, S. Alvarez, R. Gassaway, A. Biocca, S.T. Thoroddsen, A. Hexemer, A. Amassian, Spin-Cast Bulk Heterojunction Solar Cells: A Dynamical Investigation, Adv. Mater. 25 (2013) 1923-1929. doi:10.1002/adma.201203440.

[26] L.H. Rossander, N.K. Zawacka, H.F. Dam, F.C. Krebs, J.W. Andreasen, In situ 
monitoring of structure formation in the active layer of polymer solar cells during roll-toroll coating, AIP Adv. 4 (2014) 87105. doi:10.1063/1.4892526.

[27] M. Sanyal, B. Schmidt-Hansberg, M.F.G. Klein, A. Colsmann, C. Munuera, A. Vorobiev, U. Lemmer, W. Schabel, H. Dosch, E. Barrena, In Situ X-Ray Study of DryingTemperature Influence on the Structural Evolution of Bulk-Heterojunction PolymerFullerene Solar Cells Processed by Doctor-Blading, Adv. Energy Mater. 1 (2011) 363367. doi:10.1002/aenm.201100007.

[28] T. Kassar, N.S. Güldal, M. Berlinghof, T. Ameri, A. Kratzer, B.C. Schroeder, G.L. Destri, A. Hirsch, M. Heeney, I. McCulloch, C.J. Brabec, T. Unruh, Real-Time Investigation of Intercalation and Structure Evolution in Printed Polymer:Fullerene Bulk Heterojunction Thin Films, Adv. Energy Mater. 6 (2016) 1502025. doi:10.1002/aenm.201502025.

[29] R.C. Nieuwendaal, H.W. Ro, D.S. Germack, R.J. Kline, M.F. Toney, C.K. Chan, A. Agrawal, D. Gundlach, D.L. VanderHart, D.M. Delongchamp, Measuring Domain Sizes and Compositional Heterogeneities in P3HT-PCBM Bulk Heterojunction Thin Films with 1H Spin Diffusion NMR Spectroscopy, Adv. Funct. Mater. 22 (2012) 1255-1266. doi:10.1002/adfm.201102138.

[30] S. Nilsson, A. Bernasik, A. Budkowski, E. Moons, $\uparrow$ Svante Nilsson, $¥$ Andrzej Bernasik, $\S$ and Andrzej Budkowski, $\uparrow$ Ellen Moons*, Morphology and phase segregation of spincasted films of polyfluorene/PCBM blends, Macromolecules. 40 (2007) 8291-8301. doi:10.1021/ma070712a.

[31] M. Campoy-Quiles, T. Ferenczi, T. Agostinelli, P.G. Etchegoin, Y. Kim, T.D. Anthopoulos, P.N. Stavrinou, D.D.C. Bradley, J. Nelson, Morphology evolution via self- 
organization and lateral and vertical diffusion in polymer:fullerene solar cell blends, Nat. Mater. 7 (2008) 158-164. doi:10.1038/nmat2102.

[32] Y. Vaynzof, D. Kabra, L. Zhao, L.L. Chua, U. Steiner, R.H. Friend, Surface-Directed Spinodal Decomposition in Poly[3-hexylthiophene] and C ${ }_{61}$-Butyric Acid Methyl Ester Blends, ACS Nano. 5 (2011) 329-336. doi:10.1021/nn102899g.

[33] J.J. van Franeker, M. Turbiez, W. Li, M.M. Wienk, R. a. J. Janssen, A real-time study of the benefits of co-solvents in polymer solar cell processing, Nat. Commun. 6 (2015) 6229. doi:10.1038/ncomms7229.

[34] J.J. van Franeker, G.H.L. Heintges, C. Schaefer, G. Portale, W. Li, M.M. Wienk, P. van der Schoot, R.A.J. Janssen, Polymer Solar Cells: Solubility Controls Fiber Network Formation, J. Am. Chem. Soc. 137 (2015) 11783-11794. doi:10.1021/jacs.5b07228.

[35] O. Wodo, B. Ganapathysubramanian, Modeling morphology evolution during solventbased fabrication of organic solar cells, Comput. Mater. Sci. 55 (2012) 113-126. doi:10.1016/j.commatsci.2011.12.012.

[36] M. Le Berre, Y. Chen, D. Baigl, From convective assembly to Landau-Levich deposition of multilayered phospholipid films of controlled thickness., Langmuir. 25 (2009) 2554-7. doi:10.1021/la803646e.

[37] H. Matsuura, S. Iwaasa, Y. Nagasaka, Mass Diffusion Coefficient and Soret Coefficient of $o$-Dichlorobenzene Solutions of PCBM and [60]Fullerene by the Soret Forced Rayleigh Scattering Method, J. Chem. Eng. Data. 60 (2015) 3621-3630. doi:10.1021/acs.jced.5b00609. 
[38] R. Stalder, J. Mei, K.R. Graham, L. a. Estrada, J.R. Reynolds, Isoindigo, a versatile electron-deficient unit for high-performance organic electronics, Chem. Mater. 26 (2014) 664-678. doi:10.1021/cm402219v.

[39] R.-J. Roe, Methods of X-ray and neutron scattering in polymer science, 2000. http://books.google.co.uk/books?id=v5s1bEcAPEsC.

[40] C.R. McNeill, H. Ade, Soft X-ray characterisation of organic semiconductor films, J. Mater. Chem. C. 1 (2013) 187-201. doi:10.1039/C2TC00001F.

[41] S. Swaraj, C. Wang, H. Yan, B. Watts, J. Lüning, C.R. McNeill, H. Ade, Nanomorphology of bulk heterojunction photovoltaic thin films probed with resonant soft X-ray scattering, Nano Lett. 10 (2010) 2863-2869. doi:10.1021/nl1009266.

[42] J. Rivnay, S.C.B. Mannsfeld, C.E. Miller, A. Salleo, M.F. Toney, Quantitative determination of organic semiconductor microstructure from the molecular to device scale, Chem. Rev. 112 (2012) 5488-5519. doi:10.1021/cr3001109.

[43] J.L. Baker, L.H. Jimison, S. Mannsfeld, S. Volkman, S. Yin, V. Subramanian, A. Salleo, A.P. Alivisatos, M.F. Toney, Quantification of Thin Film Crystallographic Orientation Using X-ray Diffraction with an Area Detector, Langmuir. 26 (2010) 9146-9151. doi:10.1021/la904840q.

[44] M.R. Hammond, R.J. Kline, A.A. Herzing, L.J. Richter, D.S. Germack, H.-W. Ro, C.L. Soles, D. a Fischer, T. Xu, L. Yu, M.F. Toney, D.M. DeLongchamp, Molecular Order in High-Efficiency Polymer/Fullerene Bulk Heterojunction Solar Cells, ACS Nano. 5 (2011) 8248-8257. doi:10.1021/nn202951e. 
[45] B.A. Collins, J.R. Tumbleston, H. Ade, Miscibility, Crystallinity, and Phase Development in P3HT/PCBM Solar Cells: Toward an Enlightened Understanding of Device Morphology and Stability, J. Phys. Chem. Lett. 2 (2011) 3135-3145. doi:10.1021/jz2014902.

[46] B. Schmidt-Hansberg, M.F.G. Klein, K. Peters, F. Buss, J. Pfeifer, S. Walheim, A. Colsmann, U. Lemmer, P. Scharfer, W. Schabel, In situ monitoring the drying kinetics of knife coated polymer-fullerene films for organic solar cells, J. Appl. Phys. 106 (2009) 124501. doi:10.1063/1.3270402.

[47] J.T. Rogers, K. Schmidt, M.F. Toney, G.C. Bazan, E.J. Kramer, Time-resolved structural evolution of additive-processed bulk heterojunction solar cells., J. Am. Chem. Soc. 134 (2012) 2884-7. doi:10.1021/ja2104747.

[48] N.D. Treat, C.G. Shuttle, M.F. Toney, C.J. Hawker, M.L. Chabinyc, In situ measurement of power conversion efficiency and molecular ordering during thermal annealing in P3HT:PCBM bulk heterojunction solar cells, J. Mater. Chem. 21 (2011) 15224. doi:10.1039/c1jm12677f.

[49] G. Li, Y. Yao, H. Yang, V. Shrotriya, G. Yang, Y. Yang, "Solvent annealing" effect in polymer solar cells based on poly(3-hexylthiophene) and methanofullerenes, Adv. Funct. Mater. 17 (2007) 1636-1644. doi:10.1002/adfm.200600624.

[50] D.R. Lide, CRC Handbook of Chemistry and Physics, 94th Edition, 2013-2014, 2013. doi:10.1136/oem.53.7.504.

[51] Y. Zhou, Q. Yan, Y.-Q. Zheng, J.-Y. Wang, D. Zhao, J. Pei, New polymer acceptors for 
organic solar cells: the effect of regio-regularity and device configuration, J. Mater. Chem. A. 1 (2013) 6609. doi:10.1039/c3ta10864c.

[52] L. Fang, Y. Zhou, Y.-X. Yao, Y. Diao, W.-Y. Lee, A.L. Appleton, R. Allen, J. Reinspach, S.C.B. Mannsfeld, Z. Bao, Side-Chain Engineering of Isoindigo-Containing Conjugated Polymers Using Polystyrene for High-Performance Bulk Heterojunction Solar Cells, Chem. Mater. 25 (2013) 4874-4880. doi:10.1021/cm4024259.

[53] T. Lei, Y. Cao, X. Zhou, Y. Peng, J. Bian, J. Pei, Systematic investigation of isoindigobased polymeric field-effect transistors: Design strategy and impact of polymer symmetry and backbone curvature, Chem. Mater. 24 (2012) 1762-1770. doi:10.1021/cm300117x.

[54] Y. Sun, J.H. Seo, C.J. Takacs, J. Seifter, A.J. Heeger, Inverted polymer solar cells integrated with a low-temperature-annealed sol-gel-derived $\mathrm{ZnO}$ Film as an electron transport layer., Adv. Mater. 23 (2011) 1679-83. doi:10.1002/adma.201004301.

[55] E. Gann, A.T. Young, B.A. Collins, H. Yan, J. Nasiatka, H.A. Padmore, H. Ade, A. Hexemer, C. Wang, Soft x-ray scattering facility at the Advanced Light Source with realtime data processing and analysis, Rev. Sci. Instrum. 83 (2012) 45110. doi:10.1063/1.3701831. 


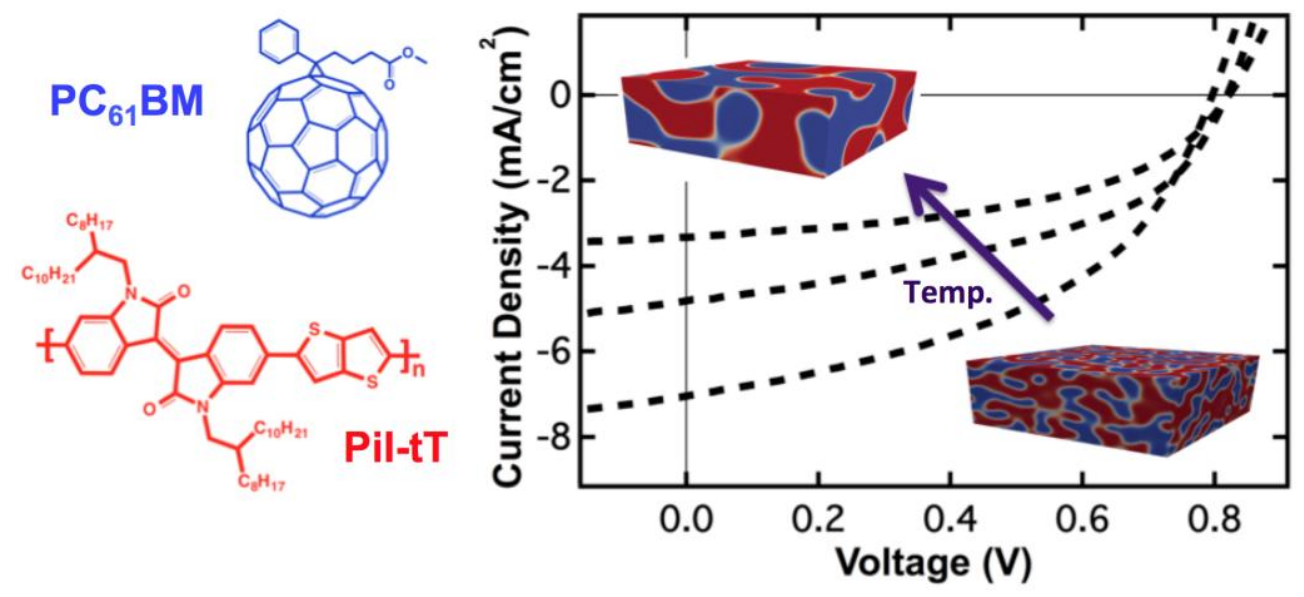

